\title{
FIXED POINT THEOREM FOR NONEXPANSIVE SEMIGROUPS ON BANACH SPACE
}

\author{
WATARU TAKAHASHI AND DOO HOAN JEONG
}

(Communicated by Palle E. T. Jorgensen)

\begin{abstract}
Let $C$ be a nonempty closed convex subset of a uniformly convex Banach space, and let $S$ be a semitopological semigroup such that $\operatorname{RUC}(S)$ has a left invariant submean. Then we prove a fixed point theorem for a continuous representation of $S$ as nonexpansive mappings on $C$.
\end{abstract}

\section{INTRODUCTION}

Let $S$ be a semitopological semigroup, i.e., $S$ is a semigroup with a Hausdorff topology such that for each $a \in S$ the mappings $s \rightarrow s a$ and $s \rightarrow a s$ from $S$ into $S$ are continuous. Let $C$ be a nonempty subset of a Banach space $E$, and let $\mathscr{F}=\left\{T_{t}: t \in S\right\}$ be a family of self-maps of $C$. Then $\mathscr{F}$ is said to be a continuous representation of $S$ as nonexpansive mappings on $C$ if the following conditions are satisfied:

(1) $T_{s t} x=T_{s} T_{t} x$ for all $t, s \in S$ and $x \in C$;

(2) the mapping $(s, x) \rightarrow T_{s} x$ from $S \times C$ into $C$ is continuous when $S \times C$ has the product topology.

Fixed point theorems for a continuous representation of $S$ as nonexpansive mappings on $C$ have been investigated by several authors; see, for example, Bartoszek [2], Lau [3], Lau and Takahashi [4, 5], Mizoguchi and Takahashi [7], Takahashi [8-10], Tan and Xu [11], Xu [12], and others. Recently, Lau and Takahashi [5] and Tan and Xu [11] proved fixed point theorems for such a representation in the case of which $E$ is a uniformly convex Banach space and $\operatorname{RUC}(S)$, the space of bounded right uniformly continuous functions on $S$, has a left invariant mean. On the other hand, Mizoguchi and Takahashi [7] introduced the notion of submean which generalizes "mean" and "limsup" and proved a fixed point theorem in a Hilbert space which generalizes simultaneously fixed point theorems for left amenable semigroups and left reversible semigroups.

Received by the editors April 1, 1993.

1991 Mathematics Subject Classification. Primary 47H10.

Key words and phrases. Fixed point, nonexpansive mapping, mean.

This paper was prepared during the second author's stay at Tokyo Institute of Technology under financial support by Korea Science and Engineering Foundation, 1991. 
In this paper, we prove a fixed point theorem for a continuous representation $\mathscr{F}$ in the case of which $E$ is a uniformly convex Banach space and $\operatorname{RUC}(S)$ has a left invariant submean. This theorem generalizes the results $[9,5,11]$.

\section{FIXED POINT THEOREM}

Let $S$ be a set, and let $m(S)$ be the Banach space of all bounded realvalued functions on $S$ with supremum norm. Let $X$ be a subspace of $m(S)$ containing constants. A real-valued function $\mu$ on $X$ is called a submean on $X$ [7] if the following conditions are satisfied:

(1) $\mu(f+g) \leq \mu(f)+\mu(g)$ for every $f, g \in X$;

(2) $\mu(\alpha f)=\alpha \mu(f)$ for every $f \in X$ and $\alpha \geq 0$;

(3) for $f, g \in X, f \leq g$ implies $\mu(f) \leq \mu(g)$;

(4) $\mu(c)=c$ for every constant $c$.

Let $\mu$ be a submean on $X$ and $f \in X$. Then, according to time and circumstances, we use $\mu_{t}(f(t))$ instead of $\mu(f)$. The following proposition is used to prove the lemma.

Proposition (cf. [12, 13]). Let $p>1$ and $b>0$ be two fixed numbers. Then a Banach space $E$ is uniformly convex if and only if there exists a continuous, strictly increasing, and convex function (depending on $p$ and $b) \quad g:[0, \infty) \rightarrow$ $[0, \infty)$ such that $g(0)=0$ and

$$
\|\lambda x+(1-\lambda) y\|^{p} \leq \lambda\|x\|^{p}+(1-\lambda)\|y\|^{p}-W_{p}(\lambda) g(\|x-y\|)
$$

for all $x, y \in B_{b}$ and $0 \leq \lambda \leq 1$, where $W_{p}(\lambda)=\lambda(1-\lambda)^{p}+\lambda^{p}(1-\lambda)$ and $B_{b}$ is the closed ball with radius $b$ and centered at the origin.

Lemma. Let $C$ be a nonempty closed convex subset of a uniformly convex $\mathrm{Ba}$ nach space, let $S$ be an index set, and let $\left\{x_{t}: t \in S\right\}$ be a bounded set of $E$. Let $X$ be a subspace of $m(S)$ containing constants, and let $\mu$ be a submean on $X$. Suppose that for each $x \in C$ the real-valued function on $S$ defined by

$$
f(t)=\left\|x_{t}-x\right\|^{2} \text { for all } t \in S
$$

belongs to $X$. If

$$
r(x)=\mu_{t}\left\|x_{t}-x\right\|^{2} \text { for all } x \in C
$$

and $r=\inf \{r(x): x \in C\}$, then there exists a unique element $z \in C$ such that $r(z)=r$.

Proof. We first prove that the real-valued function $r$ on $C$ is continuous and convex. Let $x_{n} \rightarrow x$ and

$$
M=\sup \left\{\left\|x_{t}-x_{n}\right\|+\left\|x_{t}-x\right\|: n=1,2, \ldots, t \in S\right\} .
$$

Then, since

$$
\begin{aligned}
\left\|x_{t}-x_{n}\right\|^{2}-\left\|x_{t}-x\right\|^{2} & =\left(\left\|x_{t}-x_{n}\right\|+\left\|x_{t}-x\right\|\right)\left(\left\|x_{t}-x_{n}\right\|-\left\|x_{t}-x\right\|\right) \\
& \leq M\left|\left\|x_{t}-x_{n}\right\|-\left\|x_{t}-x\right\|\right| \leq M\left\|x_{n}-x\right\|
\end{aligned}
$$

for every $n=1,2, \ldots$ and $t \in S$, we have

$$
\mu_{t}\left\|x_{t}-x_{n}\right\|^{2} \leq \mu_{t}\left\|x_{t}-x\right\|^{2}+M\left\|x_{n}-x\right\| .
$$

Similarly we have

$$
\mu_{t}\left\|x_{t}-x\right\|^{2} \leq \mu_{t}\left\|x_{t}-x_{n}\right\|^{2}+M\left\|x_{n}-x\right\| .
$$


So we have $\left|r\left(x_{n}\right)-r(x)\right| \leq M\left\|x_{n}-x\right\|$. This implies that $r$ is continuous on $C$. Let $\alpha$ and $\beta$ be nonnegative numbers with $\alpha+\beta=1$ and $x, y \in C$. Then, since

$$
\left\|x_{t}-(\alpha x+\beta y)\right\|^{2} \leq \alpha\left\|x_{t}-x\right\|^{2}+\beta\left\|x_{t}-y\right\|^{2},
$$

we have $r(\alpha x+\beta y) \leq \alpha r(x)+\beta r(y)$. This implies that $r$ is convex. We can also prove that if $\left\|x_{n}\right\| \rightarrow \infty$, then $r\left(x_{n}\right) \rightarrow \infty$. In fact, since

$$
\left\|x_{n}\right\|^{2} \leq 2\left\|x_{n}-x_{t}\right\|^{2}+2\left\|x_{t}\right\|^{2}
$$

we have

$$
\left\|x_{n}\right\|^{2} \leq 2 r\left(x_{n}\right)+2 M^{\prime},
$$

where $M^{\prime}=\sup \left\{\left\|x_{t}\right\|^{2}: t \in S\right\}$. So if $\left\|x_{n}\right\| \rightarrow \infty$, then $r\left(x_{n}\right) \rightarrow \infty$. Therefore, it follows from [1, p. 79] that there is an element $z \in C$ with $r(z)=r$. Next we show that such an element $z \in C$ is unique. Let $K=\{z \in C: r(z)=r\}$. Then it is obvious that $K$ is nonempty, closed, and convex. Further, $K$ is bounded. In fact, let $z \in K$. Then since

$$
\|z\|^{2} \leq 2\left\|z-x_{t}\right\|^{2}+2\left\|x_{t}\right\|^{2} \text { for all } t \in S,
$$

we have

$$
\|z\|^{2} \leq 2 r(z)+2 M^{\prime}=2 r+2 M^{\prime} .
$$

Choose $a>0$ large enough so that $\left\{x_{t}: t \in S\right\} \cup K \subset B_{a}$, and put $b=2 a$. Then since $x_{t}-z_{1}, x_{t}-z_{2} \in B_{b}$ for all $t \in S$ and $z_{1}, z_{2} \in K$, from the proposition, we have

$$
\left\|x_{t}-\frac{1}{2}\left(z_{1}+z_{2}\right)\right\|^{2} \leq \frac{1}{2}\left\|x_{t}-z_{1}\right\|^{2}+\frac{1}{2}\left\|x_{t}-z_{2}\right\|^{2}-\frac{1}{4} g\left(\left\|z_{1}-z_{2}\right\|\right) .
$$

So if $z_{1} \neq z_{2}$, we have

$$
r\left(\frac{1}{2}\left(z_{1}+z_{2}\right)\right) \leq \frac{1}{2} r\left(z_{1}\right)+\frac{1}{2} r\left(z_{2}\right)-\frac{1}{4} g\left(\left\|z_{1}-z_{2}\right\|\right)<r .
$$

This is a contradiction. Therefore, there exists a unique element $z \in C$ with $r(z)=r$.

Let $S$ be a semitopological semigroup. For $s \in S$ and $f \in m(S)$, we define $\left(l_{s} f\right)(t)=f(s t)$ and $\left(r_{s} f\right)(t)=f(t s)$ for all $t \in S$. Let $X$ be a subspace of $m(S)$ containing constants which is $l_{s}$-invariant, i.e., $l_{s}(X) \subset X$ for each $s \in S$. Then a submean $\mu$ on $X$ is said to be left invariant if $\mu(f)=\mu\left(l_{s} f\right)$ for all $s \in S$ and $f \in X$. Let $C(S)$ be the Banach space of bounded continuous real-valued functions on $S$. Let $\operatorname{RUC}(S)$ denote the space of bounded right uniformly continuous functions on $S$, i.e., all $f \in C(S)$ such that the mapping $s \rightarrow r_{s} f$ of $S$ into $C(S)$ is continuous. Then $\operatorname{RUC}(S)$ is a closed subalgebra of $C(S)$ containing constants and invariant under left and right translations (see [6] for details). A semitopological semigroup $S$ is left reversible if any two closed right ideals of $S$ have nonvoid intersection. In this case, $(S, \leq)$ is a directed system when the binary relation " $\leq$ " on $S$ is defined by $a \leq b$ if and only if $\{a\} \cup \overline{a S} \supset\{b\} \cup \overline{b S}$. Now we can prove a fixed point theorem for a continuous representation of $S$ such that $\operatorname{RUC}(S)$ has a left invariant submean.

Theorem. Let $S$ be a semitopological semigroup. Let $\mathscr{F}=\left\{T_{t}: t \in S\right\}$ be a continuous representation of $S$ as nonexpansive mappings on a closed convex subset $C$ of a uniformly convex Banach space $E$ into $C$. If $\operatorname{RUC}(S)$ has a left 
invariant submean $\mu$ and $C$ contains an element $u$ such that $\left\{T_{t} u: t \in S\right\}$ is bounded, then there exists $z \in C$ such that $T_{s} z=z$ for all $s \in S$.

Proof. First observe that for each $y \in C$ the function $h$ defined by

$$
h(t)=\left\|T_{t} u-y\right\|^{2} \quad \text { for all } t \in S
$$

belongs to $\operatorname{RUC}(S)$. In fact, as Lau and Takahashi [5], we have, for all $a, b \in$ $S$,

$$
\begin{aligned}
\left\|r_{a} h-r_{b} h\right\| & =\sup _{t \in S}\left|\left(r_{a} h\right)(t)-\left(r_{b} h\right)(t)\right|=\sup _{t \in S}|h(t a)-h(t b)| \\
& =\sup \left|\left\|T_{t a} u-y\right\|^{2}-\left\|T_{t b} u-y\right\|^{2}\right| \\
& \leq k \sup _{t \in S}\left\|T_{t a} u-T_{t b} u\right\| \leq k\left\|T_{a} u-T_{b} u\right\|,
\end{aligned}
$$

where $k=2 \sup _{t \in S}\left(\left\|T_{t} u\right\|+\|y\|\right)$. Then, $h \in \operatorname{RUC}(S)$.

Let $\mu$ be a left invariant submean on $\operatorname{RUC}(S)$. Then the set

$$
K=\left\{z \in C: \mu_{t}\left\|T_{t} u-z\right\|^{2}=\min _{y \in C} \mu_{t}\left\|T_{t} u-y\right\|^{2}\right\}
$$

is invariant under every $T_{s}, s \in S$. In fact, if $z \in K$, then for each $s \in S$ we have

$$
\mu_{t}\left\|T_{t} u-T_{s} z\right\|^{2}=\mu_{t}\left\|T_{s t} u-T_{s} z\right\|^{2}=\mu_{t}\left\|T_{s} T_{t} u-T_{s} z\right\|^{2} \leq \mu_{t}\left\|T_{t} u-z\right\|^{2}
$$

and hence $T_{s} z \in K$. On the other hand, by Lemma, we know that $K$ consists of one point. Therefore, this point is a common fixed point of $T_{s}, s \in S$.

The following two results, proved by the different methods, are deduced as the corollaries of Theorem.

Corollary 1 (cf. $[4,12])$. Let $C$ be a closed convex subset of a uniformly convex Banach space $E$, and let $\mathscr{F}=\left\{T_{t}: t \in S\right\}$ be a continuous representation of $S$ as nonexpansive mappings on $C$. Suppose that $\left\{T_{t} u: t \in S\right\}$ is bounded for some $u \in C$ and $\operatorname{RUC}(S)$ has a left invariant mean. Then there exists $z \in C$ such that $T_{s} z=z$ for all $s \in S$.

Proof. A left invariant mean $\mu$ on $\operatorname{RUC}(S)$ is a left invariant submean on $\operatorname{RUC}(S)$. Therefore, from Theorem, the proof is complete.

Corollary 2 (cf. [9]). Let $C$ be a closed convex subset of a uniformly convex Banach space $E$, and let $\mathscr{F}=\left\{T_{t}: t \in S\right\}$ be a continuous representation of $S$ as nonexpansive mappings on $C$. Suppose that $\left\{T_{t} u: t \in S\right\}$ is bounded for some $u \in C$ and $S$ is left reversible. Then there exists $z \in C$ such that $T_{s} z=z$ for all $s \in S$.

Proof. Defining a real-valued function $\mu$ on $\operatorname{RUC}(S)$ by

$$
\mu(f)=\limsup _{s} f(s) \text { for every } f \in \operatorname{RUC}(S),
$$

$\mu$ is a left invariant submean on $\operatorname{RUC}(S)$. By using Theorem, the proof is complete. 


\section{REFERENCES}

1. V. Barbu and Th. Precupanu, Convexity and optimization in Banach spaces, Editura Academiei R. S. R., Bucuresti, 1978.

2. W. Bartoszek, Nonexpansive actions of topological semigroups on strictly convex Banach space and fixed points, Proc. Amer. Math. Soc. 104 (1988), 809-811.

3. A. T. Lau, Semigroup of nonexpansive mappings on a Hilbert space, J. Math. Anal. Appl. 105 (1985), 514-522.

4. A. T. Lau and W. Takahashi, Weak convergence and non-linear ergodic theorems for reversible semigroups of nonexpansive mappings, Pacific J. Math. 126 (1987), 277-294.

5. __ Invariant means and semigroups of nonexpansive mappings on uniformly convex Banach spaces, J. Math. Anal. Appl. 153 (1990), 497-505.

6. T. Mitchel, Topological semigroups and fixed points, Illinois J. Math. 14 (1970), 630-641.

7. N. Mizoguchi and W. Takahashi, On the existence of fixed points and ergodic retractions for Lipschitzian semigroups in Hilbert spaces, Nonlinear Anal. TMA 14 (1990), 69-80.

8. W. Takahashi, $A$ nonlinear ergodic theorem for an amenable semigroup of nonexpansive mappings in a Hilbert space, Proc. Amer. Math. Soc. 81 (1981), 253-256.

9. $\ldots$, Fixed point theorems for families of nonexpansive mappings on unbounded sets, J. Math. Soc. Japan 36 (1984), 543-553.

10. $\ldots$ A nonlinear ergodic theorem for a reversible semigroup of nonexpansive mappings in a Hilbert space, Proc. Amer. Math. Soc. 97 (1986), 55-58.

11. K. K. Tan and H. K. Xu, Continuous representation of semitopological semigroup as nonexpansive mappings on Banach space (to appear).

12. H. K. Xu, Inequalities in Banach spaces with applications, Nonlinear Anal. TMA 16 (1991), $1127-1138$.

13. C. Zalinescu, On uniformly convex functions, J. Math. Anal. Appl. 95 (1983), 344-374.

Department of Information Science, Tokyo Institute of Technology, OH-okayama, Meguro-KU, TOKYO 152, JAPAN

Department of Liberal Arts, Dongeui Technical Junior College, Pusan 614-053, KoREA 